1 Universidade Estadual de Campinas (Unicamp) - Campinas (SP), Brasil. Departamento de Saúde Coletiva - Campinas (SP), Brasil. rosanaapgarcia@gmail.com

2 Universidade Estadual de Campinas (Unicamp) - Campinas (SP), Brasil. Departamento de Saúde Coletiva - Campinas (SP), Brasil.

slabbate@lexxa.com.br

\section{Institucionalização da Vigilância em Saúde de Campinas (SP) na perspectiva da Análise Institucional sócio-histórica}

\author{
Institutionalization of Health Surveillance in Campinas (SP) through \\ the perspective of socio-historical Institutional Analysis
}

Rosana Aparecida Garcia', Solange L'Abbate 2

RESUMO Este artigo teve como objetivo analisar o processo de institucionalização da Vigilância em Saúde em Campinas (SP), a partir do referencial teórico e metodológico da Análise Institucional, na vertente sócio-histórica. Foram realizadas entrevistas com sujeitos escolhidos por sua relevância em relação ao tema, articuladas a documentos que resgataram o contexto das políticas e modelos de saúde. Concluiu-se que apesar do importante significado da Vigilância em Saúde em Campinas, pouco se conhecia a respeito da sua trajetória, e analisar este tema na perspectiva dos sujeitos possibilitou resgatar processos ainda desconhecidos a respeito da Vigilância em Campinas.

PALAVRAS-CHAVE Saúde pública; Vigilância em saúde pública; Vigilância sanitária; Vigilância epidemiológica.

\begin{abstract}
This article aims to analyze the process of institutionalization of the Health Surveillance in Campinas (SP), taking into consideration the theoretical and methodological reference of Institutional Analysis in the socio-historical dimension. Interviews with subjects chosen by their relevance in relation to the theme were carried out and articulated to documents which rescued the context of politics and health models. It is concluded that, despite the significant importance of Health Surveillance in Campinas, little was known about its journey, and analyzing that theme through the perspective of the subjects, made it possible to rescue yet unknown processes about Health Surveillance in Campinas.
\end{abstract}

KEYWORDS Public health; Public health surveillance; Health surveillance; Epidemiological surveillance. 


\section{Introdução}

A preocupação com as epidemias e seu impacto sobre as sociedades é antiga, e a ideia do contágio remete a práticas, como isolamento de doentes (ROSEN, 1994; SCLIAR, 2002) e a quarentena de indivíduos, mercadorias e navios. Este contexto de controle dos indivíduos para contenção das doenças e do impacto no coletivo contribuiu para a construção da chamada 'vigilância', cujo termo e etimologia remetem à ideia de 'vigiar' - do latim vigilare. Corroborando os autores, Waldman (1991) reforça que o termo 'vigilância' está vinculado aos conceitos de isolamento e quarentena que surgem no final da Idade Média, consolidando-se nos séculos XVII e XVIII juntamente com o processo de urbanização.

Segundo Foucault (1982), no século XVIII, houve três experiências iniciadas na Europa que constituíram os elementos centrais das atuais práticas da 'vigilância': a medicina social na Inglaterra; a medicina urbana na França e a medicina de estado na Alemanha.

No Brasil, esse processo foi marcado por uma ideologia de proteção à saúde pública, utilizando ferramentas verticais de um modelo campanhista e pouco participativo, medidas de controle de portos nos séculos XVII e XVIII e ao desafio assumido por destacados sanitaristas, como Oswaldo Cruz, Carlos Chagas, Emílio Ribas e Adolfo Lutz, que enfrentaram as epidemias, como febre amarela, tifo, varíola, entre outras (WALDMAN, 2012).

A Constituição Federal de 1988, em um movimento instituinte de concretizar o direito social à saúde dos cidadãos como dever do Estado, instituiu o Sistema Único de Saúde (SUS), formado por uma rede regionalizada, hierarquizada e descentralizada, com direção única em cada esfera de governo e participação popular.

Essa proposta de descentralização e de uma abordagem integral nas ações e serviços de saúde encontrou sujeitos implicados no desejo de mudança, bem como outros resistentes, o que demonstra que "toda instituição vive um processo contínuo de transformação, através de oposições dialéticas" (ABRAHÃo, 2013, P. 317). Hess e Authier (1994) reforçam que quando o instituinte nega o instituído no sentido contrário à ideia de renovação/inovação, o instituinte pode ser conservador em relação ao instituído.

Apesar das diretrizes do SUS, houve um descompasso da vigilância em relação ao sistema de saúde como um todo (COSTA, 2003), uma vez que seus serviços e ações permaneceram centralizados até o século XX. Essa fragmentação e dicotomia também podem ser verificadas no âmbito da organização interna das estruturas administrativas das diferentes unidades federativas brasileiras.

Para Henriques (2009), dois tipos de discussão estiveram sempre presentes nesse processo: a delimitação das competências da vigilância no SUS - integração horizontal e como se deu a distribuição de tarefas entre as esferas federadas - integração longitudinal. Além disso, concorda-se com Costa (2003) quando afirma que o modelo institucional da vigilância desenvolvido no País foi pouco permeável aos movimentos sociais e manteve-se isolado das demais ações de saúde e de ações de outros âmbitos setoriais.

Associado a isso, a vigilância teve processos organizacionais e históricos diferentes entre seus núcleos específicos - Vigilância Epidemiológica (VE), Vigilância Sanitária (VS), Vigilância Ambiental (VA) e Vigilância da Saúde do Trabalhador (VST), e a institucionalização dos processos de trabalho foi construída com tecnologias, objetos e agentes específicos.

A despeito desses processos organizacionais e históricos distintos, a

Vigilância em Saúde passou a constituir-se em ação de cidadania, fundamentada na união das vigilâncias sanitária, epidemiológica, e posteriormente na Vigilância da Saúde do Trabalhador, com base no enfoque de risco e na epidemiologia social (BALISTA; SANTIAGO; CORREA FILHO, 2011, P. 760). 
Segundo a autora, essa mudança vem acompanhada da "modificação do modo de fazer vigilância, procurando a integralidade das ações".

Neste trabalho, optou-se por utilizar o referencial teórico da Análise Institucional (AI) em sua vertente sócio-histórica desenvolvida por Savoye $(1988,2003,2007)$, por entender a potência desse referencial para a abordagem da relação entre Estado e sociedade, bem como sua contribuição para uma análise do momento fundador da instituição vigilância. Segundo Savoye (2003, P. 134), a AI sócio-histórica "significa colocar em perspectiva histórica uma realidade estudada em sua atualidade".

Sól $(2011,2013)$ utilizou-se dessa vertente ao analisar três programas de residência em Medicina Geral Comunitária: dois em Porto Alegre (RS) e um em Mariana (MG), contribuindo assim para uma reflexão acerca das gêneses teórica e social desses projetos, as quais, de acordo com Savoye (2003, P. 2), constituem uma conjugação "de fatores de natureza política, social e institucional que presidem à emergência da nova práxis cognitiva”.

A partir dessa mesma conjugação, este artigo busca analisar as gêneses teórica, histórica e social da Vigilância em Saúde, em alguns de seus núcleos específicos Vigilância Epidemiológica e Vigilância Sanitária - no contexto internacional e brasileiro, dialogando com a experiência do município de Campinas (SP), e a gênese teórica que fundamentou os processos de reorganização dos sistemas de vigilância.

$\mathrm{O}$ atual contexto de mudanças sócio/histórico/econômicas mundiais tem exigido, entre outras coisas, a proteção à saúde dos sujeitos, por isso a relevância do tema proposto neste artigo.

No Brasil, a Vigilância em Saúde tem sido objeto de medidas político-institucionais do setor saúde nos níveis federal, estadual e municipal. Ao mesmo tempo, o tema da vigilância vem despertando interesse de vários autores, dentre os quais, destacam-se: Costa (2003), Aith e Dallari (2009), Henriques (2009) e Lucchese (2001), os quais têm se debruçado sobre esse objeto e o analisado a partir de diferentes vinculações teórico-metodológicas.

Este artigo é parte da tese de doutorado da primeira autora, cujo projeto foi aprovado pelo Comitê de Ética em Pesquisa da Pontifícia Universidade Católica de Campinas, em 2 de abril de 2013, registrado sob o número CAAE 14109413.6.0000.5481.

Seu principal objetivo é o de analisar o processo de institucionalização da Vigilância em Saúde em Campinas, enfocando as estratégias de centralização e descentralização, identificando a percepção dos sujeitos envolvidos sobre o processo.

\section{Percurso teórico- metodológico}

A análise deste texto fundamenta-se no referencial teórico da AI que teve origem na França, na década de 1960, foi difundida no Brasil a partir de 1970 e que "nasceu da articulação entre intervenção e pesquisa, entre teoria e prática", tendo como objetivo "compreender uma determinada realidade social e organizacional, a partir dos discursos e práticas dos seus sujeitos” (L'ABBATE, 2012, P. 198).

A autora sinaliza que a AI se utiliza de um método constituído de um conjunto articulado de conceitos, tendo como base um conceito dialético de instituição desenvolvido por Lourau a partir da lógica hegeliana e apoiado em Castoriadis. Para Lourau (1975, P. 9), toda instituição é o resultado da articulação dinâmica entre três momentos: o instituído, o instituinte e a institucionalização, e que "formas sociais visíveis, porquanto dotadas de uma organização jurídica e/ou material [...] são chamadas de instituição".

A Vigilância em Saúde é aqui entendida como uma instituição, pois possui suas normas, protocolos, legislação, saberes e 
práticas instituídas, dinamicamente questionadas em movimentos instituintes de mudanças que se dão em conflito permanente com o instituído. Nesse movimento diacrônico, dá-se o processo de institucionalização, que implica em "duração, temporalidade e historicidade" (SAVOYE, 2007, P. 181).

$O$ confronto permanente entre o instituído (o que se procura manter) e o instituinte (forças de subversão e de mudança) na vigilância resulta em seu processo de institucionalização com características contraditórias, muitas vezes capturados por aquilo que é hegemônico e está instituído (LOURAU, 1995; KAES, 1991).

Além disso, o referencial teórico da AI, por seu caráter eminentemente qualitativo, considera a relação sujeito-objeto como pertencente à mesma natureza histórica e social. Dessa forma, tomou-se como pressupostos que a opção metodológica, as questões e os objetivos da investigação são influenciados pela posição do pesquisador, ou seja, sua implicação com o objeto de estudo e análise.

A posição circunstancial ocupada pela primeira autora na coordenação da Vigilância em Saúde de um Distrito de Saúde de Campinas, e sua implicação com o tema, motivou o interesse no objeto investigado, contrapondo-se, ao mesmo tempo, à ideia da neutralidade (MINAYO, 2007), advinda do positivismo. Nesse sentido, Lourau (2004) desenvolveu o conceito de implicação, rejeitando a separação rígida entre o sujeito que conhece e o objeto a ser conhecido e a neutralidade do pesquisador. Segundo o autor, os pesquisadores são, o tempo todo, movidos pelas suas escolhas afetivas, ideológicas e profissionais, com relação à sua prática de pesquisa e/ou de intervenção.

Passos e Barros (2000, P. 73) afirmam que

a implicação não é uma questão de vontade [...] inclui uma análise do sistema de lugares, o assinalamento do lugar que ocupa que busca ocupar, e do que lhe é designado ocupar, com os riscos que isto implica
Dessa forma, o lugar ocupado pela primeira autora influenciou a análise do objeto investigado.

O local da pesquisa foi o município de Campinas (SP), que tem se destacado devido ao acúmulo de proposições inovadoras adotadas desde os anos de 1980, as quais têm marcado fortemente a política de saúde adotada. Esse protagonismo na elaboração de políticas de saúde tem sido tema de vários estudos de autores, como Balista, Santiago e Correa Filho (2011), Vilela (2005), entre outros. Apesar disso, há pouca produção acerca do processo de institucionalização da Vigilância em Saúde no município de Campinas.

No sentido de complementar a visão da referida pesquisadora sobre o processo histórico-social da Vigilância em Saúde de Campinas, foram realizadas 17 entrevistas semiestruturadas com gestores e trabalhadores que atuaram na vigilância, selecionados a partir das suas trajetórias político/ institucionais, no sentido de contribuir para conhecer melhor a 'institucionalização fundadora' da Vigilância em Saúde do município, assim como esclarecer o processo das mudanças que se fizeram necessárias e que resultaram na 'institucionalização permanente' da instituição vigilância (SAVOYE, 2007).

As entrevistas foram gravadas e transcritas com a permissão dos entrevistados por meio do Termo de Consentimento Livre e Esclarecido.

Optou-se, nas entrevistas, por realizar um recorte temporal, a partir do final da década de 1970, por ter sido este o período de institucionalização da Vigilância Epidemiológica e Vigilância Sanitária no Brasil, além do grande investimento na Atenção Básica em saúde e implantação do modelo da Medicina Comunitária e da ampliação do número das unidades de saúde no município de Campinas (L'ABBATE, 2009).

Apenas com a intenção de complementar a análise, alguns relatos das entrevistas serão abordados no item seguinte, integrados ao texto. 


\section{Resultados e discussões}

\section{Processo de institucionalização da Vigilância em Saúde no Brasil}

A opção pela organização descentralizada e integração dos serviços de saúde esteve presente nas proposições das Ações Integradas de Saúde (AIS) (1983-1987), no Sistema Unificado e Descentralizado de Saúde (Suds) (1988-1989), e a partir de 1990, no SUS, instituído pela Constituição Federal (1988).

$\mathrm{O}$ descompasso da vigilância em relação às proposições políticas nacionais pode ser relacionado, na perspectiva da $\mathrm{AI}$, ao movimento dialético entre o instituído e o instituinte, colocando em dúvida a fundação do instituído, negando-o e/ou buscando sua manutenção. Isso pode ser constatado quando se observa que as ações de vigilância foram descentralizadas e distribuídas entre a esfera federal e estadual, sem considerar as competências dos municípios.

Segundo Waldman (1991), a designação de Vigilância Epidemiológica (VE) foi consagrada internacionalmente, na década de 1970, com a criação da Unidade de Vigilância Epidemiológica da Divisão de Doenças Transmissíveis da Organização Mundial da Saúde (OMS), que incentivou os países em desenvolvimento a criarem seus sistemas de vigilância epidemiológica, tendo como objetivo, principalmente, a redução da morbimortalidade. No Brasil, sua criação se deu a partir do Programa Nacional de Imunizações (PNI), instituindo normas relativas à notificação compulsória de doenças e definindo as linhas gerais desse subsistema da Vigilância em Saúde.

A Vigilância Sanitária (VS), enquanto denominação consagrada, foi introduzida no Brasil em meados da década de 1970, em resposta à ordem econômica / política / institucional / social e técnico-científica do País, não apresentando, no entanto, uma estruturação sistêmica (LUCCHESE, 2001). A década de 1980 foi marcada pela necessidade de organizar as ações de Vigilância Sanitária por meio de um sistema nacional - Secretaria Nacional de Vigilância Sanitária (SNVS) do Ministério da Saúde (MS), que incorporou o antigo Serviço de Fiscalização da Medicina e Farmácia e o Laboratório Central de Controle de Drogas, Medicamentos e Alimentos.

Segundo Lucchese (2001), na década de 1990, em meio à influência de ideias liberalizantes, a SNVS perde a designação 'nacional' e passa a ser chamada de Secretaria de Vigilância Sanitária/MS. No final dessa mesma década, em meio a um contexto de suspeitas de corrupção e escândalos ocorridos em 1996, 1997 e 1998 na área de medicamentos, há uma reformulação organizacional instituindo uma agência reguladora nacional, a Agência Nacional de Vigilância Sanitária (Anvisa), para coordenar o recém-criado Sistema Nacional de Vigilância Sanitária (SNVS) e promover a proteção da saúde da população por intermédio do controle sanitário da produção e da comercialização de produtos e serviços submetidos à VS, bem como o controle dos portos, aeroportos e fronteiras.

Esses processos institucionais e organizacionais dentro do MS e da Anvisa estabeleceram certa dicotomia entre a VS e a VE como se fossem 'duas vigilâncias' (COSTA, 2003), posto que fossem localizadas em espaços institucionais distintos, com legislações específicas, bem como ferramentas e dispositivos, o que dificultava o trabalho em rede.

Apesar de as diretrizes do SUS apontarem para uma concepção ampliada de Vigilância em Saúde, a lógica jurídica e a fragmentação entre os núcleos - Vigilância Epidemiológica, Sanitária, Saúde do Trabalhador e Ambiental - impactaram na organização dos serviços de Vigilância em Saúde, que passaram a operar por campos de especialidade específicos, mantendo as reproduções do modelo fragmentado (VILELA, 2005).

Aith e Dallari (2009) reconhecem muitos avanços na estruturação dos serviços de 
Vigilância em Saúde no País, bem como a necessidade de muitos ajustes a serem feitos, entre eles, a necessidade de unificar os diversos sistemas de vigilância, capazes de reunir as informações necessárias para a identificação de riscos em saúde.

Um dos desafios para a Vigilância em Saúde nos dias atuais é o reconhecimento da tendência mundial de um processo de transição demográfica, epidemiológica e nutricional desde a década de 1960, que, segundo Malta e Silva Júnior (2013), resultou em uma nova epidemia mundial: o aumento da prevalência de Doenças Crônicas Não Transmissíveis (DCNT).

\section{Processo de institucionalização do Sistema Municipal de Vigilância em Saúde de Campinas (SP)}

Observando o contexto no qual a Vigilância em Saúde foi instituída no Brasil, o município de Campinas protagonizou movimentos relacionados à municipalização e descentralização das ações de Vigilância em Saúde, cuja prática foi consolidada e teve início antes da implantação do SUS e da Lei 8.080/90.

Enquanto no contexto nacional, no final da década de 1970, a organização do modelo de saúde no País caracterizava-se por apresentar tendência fortemente centralizadora, Campinas institucionalizava um modelo fundamentado na Medicina Comunitária e na participação popular, tendo como ponto de partida a proposição de uma organização descentralizada, ampliando o número das unidades de saúde, que era composto por 16 'postos de saúde' em regiões periféricas da cidade (L'ABBATE, 2009).

A partir de 1989, houve a municipalização dos Centros de Saúde do estado, e os antigos 'postos de saúde' do município ampliaram a complexidade das ações em saúde desenvolvidas com a descentralização da VE para a rede de serviços de saúde em Campinas, responsabilizando-os pela realização de um conjunto de atividades, como a notificação e investigação de casos, surtos e agravos inusitados, passando a ser denominados de Centros de Saúde. Até então, a vacinação, os tratamentos de tuberculose e de hanseníase, entre outras ações, eram realizadas somente nos Centros de Saúde do estado.

Segundo Vilela (2005, P. 37),

as ações de vigilância foram hierarquizadas em um sistema municipal de vigilância em saúde, com responsabilidades definidas para os níveis centrais, regionais e locais.

Enquanto em nível federal a atual Constituição Federal em 1988 instituiu o SUS e suas diretrizes, um grupo interinstitucional ligado à então Comissão Interinstitucional Municipal de Saúde (Cims) protagonizou um movimento instituinte que culminou com a criação do Grupo Municipal de Vigilância Epidemiológica (GMVE) em 1988, com recursos das Secretarias Estadual e Municipal de Saúde, passando a funcionar juntamente com a sede regional estadual do então Suds e contando com um médico, um enfermeiro e dois visitadores sanitários (ABRAHÃO, 2014).

À época, a Secretaria Municipal de Saúde de Campinas possuía uma área denominada Divisão de Controle de Meio Ambiente (DCMA), que era composta pelo Serviço Médico-Veterinário e de Controle de Zoonoses, Serviço de Fiscalização Sanitária e Serviço de Fiscalização da Alimentação Pública. Segundo Abrahão (2014), esses serviços operavam com pouca integração interna e nenhuma articulação com a rede de serviços de saúde. Em 1989, a Secretaria de Saúde de Campinas assumiu o desafio de integrar a Divisão de Controle de Meio Ambiente ao Grupo Municipal de Vigilância Epidemiológica.

Toda instituição vive um processo contínuo de transformação por meio de forças dialéticas, e apesar do acúmulo existente em Campinas por intermédio dos Serviços Médico-Veterinário e de Controle de Zoonoses, de Fiscalização Sanitária e de Fiscalização da Alimentação Pública, a 
municipalização da VS de bens e serviços de interesse à saúde, como produtos de beleza, limpeza, saneantes, médico-cirúrgicos, entre outros, foi realizada em meio a contínuas contradições.

Segundo os entrevistados, foi uma descentralização solitária, sem infraestrutura, sem preparo técnico (capacitação) dos trabalhadores e com uma equipe reduzida para as ações a serem desenvolvidas. Os entrevistados referiram que não havia integração interna, o que reforçava a dicotomia entre as áreas de fiscalização. Outro limite citado pelos entrevistados foi de uma maior valorização da VE em detrimento de outras áreas da vigilância, devido à proximidade que esta tinha com a rede de serviços de saúde.

Em 1993, em um contexto de troca de governo municipal, uma diretriz municipal de descentralização político-administrativa e da horizontalização das estruturas gerenciais traz um movimento instituinte importante para o conceito Vigilância em Saúde. Foi um período de priorização de projetos intersetoriais em que a estratégia utilizada foi a criação de quatro Secretarias de Ação Regionais (SAR) - Norte, Sul, Leste e Oeste -, que tinham a responsabilidade de gerenciamento, planejamento e execução dos serviços possíveis de serem descentralizados.

Segundo os entrevistados, apesar de esse ter sido um momento de mudança rápida e pouco participativa, e 'cheia de atropelamentos', causando receios, foi reconhecido como um momento de concreta 'vivência' da Vigilância em Saúde, em seu conceito ampliado, transcendendo o campo da saúde e expandindo para outros setores e órgãos governamentais ou não. Pode-se observar nos relatos de algumas entrevistas que, apesar das contradições e reações quanto às mudanças, no espaço instituído debateram-se propostas instituintes, as quais sobrevivem em toda instituição mediante o desejo transformador dos seus sujeitos.

A partir dessa organização e responsabilização territorial, foram realizados importantes enfrentamentos de grandes epidemias, como da meningite e do sarampo (1996), da dengue (1998) e, posteriormente, da febre amarela (2000). Os Centros de Saúde, por intermédio de suas respectivas áreas de abrangência, tornaram-se responsáveis por notificações e investigações de surtos e agravos inusitados, incluindo as medidas de controle.

Em 1994, houve a implantação descentralizada do Sistema Nacional de Agravos Notificáveis (Sinan) nos serviços de Vigilância em Saúde (Visa), e foram criadas cinco Vigilâncias Distritais - Leste, Sul, Norte, Noroeste e Sudoeste - para servir como apoio técnico às equipes de saúde e fazer a gestão do sistema de vigilância das unidades de seu território de abrangência.

Segundo Abrahão (2014), a área de saúde do trabalhador foi constituída no município desde 1988, quando houve a proposta do Programa de Saúde do Trabalhador (PST) por iniciativa de sindicatos, da universidade (Unicamp) e do governo municipal. Em 2001, foram descentralizadas as ações de Saúde do Trabalhador (BALISTA, ET AL., 2011) para as cinco Visas Distritais, ações que estavam centralizadas no Centro de Referência em Saúde do Trabalhador (Cerest).

Em 2001, com a mudança de governo municipal, surge uma nova proposta de reorganização do modelo de atenção à saúde em Campinas, a partir da reformulação da rede básica enquanto espaço de reorientação do novo modelo, tendo como objetivo melhorar o vínculo, garantindo o acesso e a atenção prestada aos usuários do SUS. Foi uma época avaliada pelos entrevistados como intensa e rica nas proposições, ao mesmo tempo que havia pouca participação nas decisões, apesar de muitos espaços coletivos.

Foi um momento no qual foram implantados vários arranjos institucionais, entre eles o Núcleo de Saúde Coletiva (VILELA, 2005) e o matriciamento das equipes de referência (OLIVEIRA, 2008; CAMPOS, 1999), trazendo o que Hess e Authier (1994) chamaram de questionamento das verdades que fundaram o instituído. 
Foram realizados seminários e oficinas, articulados ao novo modelo Paidéia (CAMPOS, 2003) e seus arranjos institucionais, questionando as práticas utilizadas pela Vigilância em Saúde do município de Campinas, descrevendo-as como autoritárias e assinalando a dificuldade na integração e articulação das Vigilâncias Distritais aos Distritos de Saúde e ao nível central.

Nos dias atuais, passados mais de 20 anos de descentralização da Vigilância em Saúde de Campinas, muitas mudanças ocorreram na cidade, que se constituiu em um polo metropolitano, com grande circulação de pessoas e produtos, intensa atividade comercial e industrial, abrigando uma grande rede hoteleira, um aeroporto internacional, universidades, entre outros. A mudança do perfil da saúde/doença da população também gerou demandas e o aumento na complexidade das ações em saúde dos serviços de saúde.

Na IX Conferência Municipal de Saúde (2011), foram debatidas as questões acima apresentadas, considerando a complexidade do sistema de saúde de Campinas, e a necessidade de resposta às novas necessidades apontadas pela sociedade fizeram surgir novos movimentos dentro do Sistema Municipal de Vigilância em Saúde.

Os debates que se seguiram em 2012/2013 tiveram como objetivo revisar o processo de descentralização da Vigilância em Saúde, considerando a necessidade de trabalhar na lógica de responsabilidades compartilhadas, ou seja, as Vigilâncias Distritais - hoje denominadas de Vigilâncias Regionais -, assumindo um conjunto maior de ações de investigação e controle de doenças transmissíveis em erradicação, aquelas com baixos coeficientes de incidência (doença meningocócica) e aquelas que ocorrem de maneira espacialmente agregadas (febre maculosa, leishmanioses e leptospiroses). Segundo os entrevistados, essas ações exigem uma experiência que os serviços de saúde não desenvolveram devido à esporadicidade na qual acontecem em seu cotidiano.

\section{Os desafios atuais da Vigilância em Saúde de Campinas e do Brasil}

Como no contexto nacional, a Vigilância em Saúde de Campinas também possui o desafio de articular suas ações em rede, apesar de serem inegáveis os movimentos instituintes existentes, a partir de sujeitos implicados na superação das adversidades.

Os diferentes processos sócio-histórico-políticos nos quais se deu a institucionalização do Sistema de Vigilância em Saúde de Campinas contribuíram, no entanto, para ações que pouco se articulam internamente entre os seus núcleos específicos - Vigilância Sanitária, Vigilância Epidemiológica, Vigilância Ambiental e Vigilância em Saúde do Trabalhador - o que Henriques (2009) chamou de 'integração horizontal'.

Dentro do desafio da articulação da Vigilância em Saúde em um trabalho em rede, são emergentes novos processos de trabalho e um novo olhar para o território, coabitando com uma tensão dialética com o instituído, muitas vezes argumentando formas diferentes de fazer, mas que traduzem o mesmo regime de construção. É importante ressaltar que um novo olhar sobre o território pressupõe uma nova postura, novos processos de trabalho e de gestão enquanto instituição: é o fazer compartilhado e 'junto com', e não somente um "verniz diferente" (ABRAHÃO, 2013, P. 320).

A aproximação da vigilância aos outros serviços de saúde no território pressupõe

novas possibilidades de trocas de saber entre os profissionais de saúde em diversos níveis de atenção, favorecendo, também, maior articulação e qualificação da rede de serviços que compõe o sistema de saúde. (OLIVEIRA, 2008, P. 273).

Outro desafio a ser enfrentando é o resgate do significado das ferramentas utilizadas para gerar informação na vigilância - como o 
Sinan, o Sistema de Informação do Programa Nacional de Imunização (SIPNI), o Sistema de controle de pacientes com tuberculose (TB-Web), entre outros.

As fichas de investigação epidemiológica têm se transformado em instrumentos meramente burocráticos, preenchidas muitas vezes de forma inadequada pelas equipes de saúde, e uma possível explicação para o fato pode ser a falta de retorno das informações em tempo ágil para as intervenções.

Além disso, os clássicos (e importantes) bancos de dados estão baseados em doenças e exigem que haja uma articulação do sujeito dentro de uma linha de cuidado, e não a partir de rótulos, como 'caso de tuberculose', 'atraso vacinal', 'paciente com dengue', entre outros.

A partir desse novo olhar, seriam considerados os limites que levam os serviços de saúde a ter dificuldades em dar o retorno ágil esperado pela vigilância, no tempo hábil de alimentação dos sistemas de informação em saúde. Seria o "aprender fazendo e produzir aprendendo, modificando seu próprio modo de estar no mundo e no trabalho" (OLIVEIRA, 2008, P. 274).

Os limites são os clássicos tempos que a vigilância tem para alimentar os Sistemas de Informação em Saúde, e que muitas vezes não é o tempo da vida dos usuários e das equipes. Entra-se então em conflitos, como, por exemplo, os atrasos em alimentar os bancos que são cobrados pelos entes federados e que devem ser enfrentados na perspectiva de uma nova concepção de fazer vigilância: vigilância do que, para o que e para quem. Se isso não for enfrentando, corre-se o risco de se manterem as práticas verticais e autoritárias, utilizadas pela saúde pública e pela vigilância.

\section{Referências}

ABRAHÃO, A. L. Arranjos conceituais para a gestão em saúde a partir da Análise Institucional: relação entre gestão e subjetividade nas equipes de saúde. In:

\section{Conclusões}

Esta análise demonstra que, passados mais de 20 anos de criação do SUS, há vários desafios a serem enfrentados, entre eles, a recomposição da integralidade do objeto da Vigilância, o fortalecimento político dessa instância no cenário do MS e o estabelecimento de uma gestão participativa.

As reflexões trazidas neste estudo podem contribuir, de forma significativa, para a revisão das práticas e ferramentas utilizadas pela Vigilância em Saúde, de maneira a enfrentar novos desafios existentes na atual complexidade da sociedade e dos serviços de saúde.

Este reconhecimento remete a buscar não somente novas formas de fazer, como também um novo conceito de vigilância, tendo como pressupostos as responsabilidades compartilhadas, respeitando a autonomia dos sujeitos e o território por eles ocupado.

Esta revisão do modelo de vigilância preconiza um trabalho em rede e o investimento nos profissionais inseridos no interior da Instituição Vigilância, para que desenvolvam um olhar não focado somente em 'doenças que alimentam bancos de dados', e sim em pessoas com sofrimentos e submetidas a situações adversas com adoecimento que deve ser monitorado pela vigilância 'junto com' os serviços de saúde.

A despeito das dificuldades enfrentadas nos processos de descentralização, a experiência vivenciada em Campinas demonstrou avanços na aproximação da Vigilância em Saúde com os outros serviços de saúde e à realidade dos usuários.
L'ABBATE, S. MOURÃO, L. C.; PEZZATO, L. M. (Org.). Análise Institucional \&t Saúde Coletiva. São Paulo: Hucitec, 2013, p. 315-332. 
ABRAHÃO, C. E. C. Municipalização, descentralização e vigilância à saúde em Campinas, 1988-1994. Campinas. [internet]. Disponível em: <http://www.carlosecabrahao.med.br/saude/monografias/mono00004.htm>. Acesso em: 15 out. 2014.

AITH, F.; DALLARI, S. G. Vigilância em Saúde no Brasil: os desafios dos riscos sanitários do século XXI e a necessidade de criação de um Sistema Nacional de Vigilância em Saúde. Revista de Direito Sanitário, São Paulo, v. 10, n. 2, p. 94-125, jul./out. 2009.

BALISTA, S. R. R.; SANTIAGO, S. M.; CORREA

FILHO, H. R. A descentralização da vigilância da saúde do trabalhador no Município de Campinas, São Paulo, Brasil: uma avaliação do processo. Cadernos de Saúde Pública, Rio de Janeiro, v. 27, n. 4, p. 759-768. 2011.

BRASIL. Constituição (1988). Constituição da República Federativa. Brasília, DF: Senado Federal, 1988.

CAMPINAS. Prefeitura Municipal de Campinas. Secretaria Municipal de Saúde. Seminário Municipal de Saúde Coletiva: construção coletiva da linha do tempo. São Paulo: SMS, 2004.

CAMPOS, G. W. S. Saúde Paidéia. 2. ed. São Paulo: Hucitec, 2003.

Equipes de referência e apoio especializado matricial: um ensaio sobre a reorganização do trabalho em saúde. Ciência \&t Saúde Coletiva, Rio de Janeiro, v. 4, n. 2, p. 393-403, 1999. Disponível em: <http://www.scielo.br/pdf/csc/v4n2/7121.pdf>. Acesso em: 14 out. 2014.

COSTA, E. A. Vigilância Sanitária e proteção da saúde. Direito e Saúde Pública: coletânea de textos. Brasília, DF: Ministério da Saúde, v.1, p. 180-203, 2003. (Série E Legislação e Saúde).

FOUCAULT, M. O nascimento da medicina social. In: A Microfísica do Poder. 3. ed. Rio de Janeiro: Graal, 1982, p. 79-80.
HENRIQUES, C. M. P. As diferentes visões filosóficas e abordagens científicas sobre a Vigilância em Saúde no Brasil. Revista de Direito Sanitário. São Paulo, v. 10, n. 2, p. 87-93, jul./out. 2009.

HESS, R.; AUTHIER, M. L'instituant et l'institué. In: L'analyse institutionnelle. Paris: PUF, 1994, p. 38-49.

HESS, R. Do efeito Mühlmann ao princípio de falsificação: instituinte, instituído, institucionalização. Mnemosine, Rio de Janeiro, v. 3, n. 2, p. 148-163. 2007. Disponível em: $<$ http://www.mnemosine.com.br/ojs/index.php/mnemosine/article/view/120/pdf_109>. Acesso em: 15 out. 2014.

KAES, R. Realidade psíquica e sofrimento nas instituições. In: KAES, R. et al. (Org.). A instituição e as instituições. São Paulo: Casa do Psicólogo, 1991, p. 1-39.

L'ABBATE, S. Analise Institucional e intervenção: breve referência à gênese social e histórica de uma articulação e sua aplicação na Saúde Coletiva. Mnemosine, Rio de Janeiro, v. 8, n. 1, p. 194-219, 2012.

Direito à saúde: discursos e práticas na construção do SUS. São Paulo: Hucitec, 2010.

Análise Institucional e Saúde Coletiva. Ciência e Saúde Coletiva, Rio de Janeiro, v. 8, n. 1, p. 265-274. 2003.

LOURAU, R. A Análise Institucional. Petrópolis: Vozes, 1975.

A Análise Institucional. 2. ed. Petrópolis: Vozes, 1995.

. René Lourau na UERJ. Análise Institucional e Práticas de Pesquisa. Rio de Janeiro, UERJ, 1993. Mnemosine, Rio de Janeiro, v. 3, n. 2, p. 77-92. 2007. Implicação: um novo paradigma? In: ALTOÉ, S. (Org.). René Lourau: analista institucional em tempo integral. São Paulo: Hucitec, 2004, p. 246-258.

LUCCHESE, G. Globalização e Regulação Sanitária - rumos da vigilância sanitária no Brasil. 2001. 329 
f. Tese (Doutorado em Saúde Pública) - Fundação

Oswaldo Cruz, Rio de Janeiro, 2001.

MALTA, D. C.; SILVA JÚNIOR, J. B. O Plano de Ações Estratégicas para o Enfrentamento das Doenças Crônicas Não Transmissíveis no Brasil e a definição das metas globais para o enfrentamento dessas doenças até 2025: uma revisão. Epidemiologia e Serviços de Saúde, Brasília, DF, v. 22, n. 1, p. 151-164, 2013.

MINAYO, M. C. S. O desafio do conhecimento: pesquisa qualitativa em saúde. São Paulo: Hucitec, 2007.

OLIVEIRA, G. N. Apoio Matricial como tecnologia de gestão e articulação em rede. In: CAMPOS, G. W. S.; GUERRERO, A. V. P. (Org.). Manual de Práticas de Atenção Básica: saúde ampliada e compartilhada. São Paulo: Hucitec, 2008, p. 273-282.

PASSOS, E.; BARROS, R. B. A construção do plano da clínica e o conceito de transdisciplinaridade. Psicologia: Teoria e Pesquisa, Brasília, DF, v. 16, n. 1, p. 71-79, jan./abr. 2000.

ROSEN, G. Uma História da Saúde Pública. São Paulo: Hucitec, 1994.

SAVOYE, A. Análise Institucional e pesquisas sócio históricas: estado atual e novas perspectivas. Mnemosine, Rio de Janeiro, v. 3, n. 2, p. 181-193, 2007. Disponível em: <www.cliopsyche.cjb.net/mnemo/index.php/mnemo/ issue/view/20>. Acesso em: 15 out. 2014.

Analyse Institutionnelle et recherche sócio-historiques: quele compatibilité? L'Homme et la Société, Paris, n. 147-148, p. 2; 133-150, 2003.

Du passé faisons l'analyses. Le traitement de l'histoire. In: SAVOYE, A.; HESS, R. (Org.). Perspectives de l'Analyse Institutionnelle. Paris: Méridiens Klincksieck, 1988, p. 153-164.
SCLIAR, M. Do mágico ao social: trajetória da saúde pública. São Paulo: Senac, 2002.

SILVA JUNIOR, J. B. As doenças transmissíveis no Brasil: tendências e novos desafios para o Sistema Único de Saúde. Brasil. Ministério da Saúde. In: Saúde Brasil 2008: 20 anos de Sistema Único de Saúde (SUS) no Brasil. Brasília, DF: Ministério da Saúde, 2009.

SÓL, N. A. A. A Medicina Geral Comunitária no Brasil: uma análise institucional sócio histórica de sua trajetória enfocando programas específicos. 2011. $252 \mathrm{f}$. Tese (Doutorado em Saúde Coletiva) - Universidade Estadual de Campinas, Campinas, 2011.

Medicina geral comunitária no Brasil: uma análise institucional sócio-histórica da sua trajetória. In: L’ABBATE, S.; MOURÃO, L. C.; PEZZATO, L. M. (Org.). Análise Institucional \&t Saúde Coletiva. São Paulo: Hucitec, p. 180-205, 2013.

VILELA, M. F. G. Da 'moça da vigilância' ao núcleo de Saúde Coletiva na unidade básica de saúde: o que há de novo no modelo assistencial de Campinas. $224 \mathrm{f}$. Tese (Doutorado em Saúde Coletiva) - Universidade Estadual de Campinas, Campinas, 2005.

WALDMAN, E. A. Os 110 anos de Vigilância em Saúde no Brasil. Epidemiologia e Serviços de Saúde, Brasília, v. 21, n. 3, p. 365-366, jul./set. 2012.

A vigilância epidemiológica como prática de Saúde Pública. 1991. 228 f. Tese (Doutorado em Saúde Pública) - Universidade de São Paulo, São Paulo, 1991.

Recebido para publicação em outubro de 2014

Versão final em maio de 2015

Conflito de interesse: inexistente

Suporte financeiro: não houve 